\title{
Prevalence of Fusarium oxysporum f. sp. ciceris Causing Wilt in Chickpea and Its Pathogenic, Cultural and Morphological Characterization
}

\author{
P. Murali Sankar ${ }^{1 *}$, S. Vanitha ${ }^{1}$, A. Kamalakannan ${ }^{1}$, \\ P. Anantha Raju ${ }^{2}$ and P. Jeyakumar ${ }^{3}$ \\ ${ }^{1}$ Department of Plant Pathology, ${ }^{2}$ Department of Pulses, ${ }^{3}$ Department of Crop Physiology, \\ Tamil Nadu Agricultural University, Coimbatore-641003 India \\ *Corresponding author
}

A B S T R A C T

Chickpea (Cicer arietinum L.) contributes $18 \%$ of the global production of grain legume and provides as an important source of dietary protein for living things. The area and production of chickpea has been reduced due to several abiotic and biotic factors. Among

Keywords

Survey, Incidence,

Micro conidia,

Macro conidia,

Pathogenic ability

Article Info

Accepted:

15 January 2018

Available Online:

10 February 2018 them soil borne pathogen of $F$. oxysporum $\mathrm{f}$. sp. ciceris causing severe yield loss now a days. In this study conducted for a prevalence of wilt incidence percentage varied from 34.00 to 57.33 per cent in chickpea due to $F$. oxysporum f. sp. ciceris in Tamil Nadu. Continuously the pathogenic ability, cultural and morphological characterization was carried out. Among the fifteen isolates Foc4 (Gomangalampudur) is highly pathogenic when compared to other and causing early wilt in JAKI-9218. Grouping of isolates based on their virulence potential isolates like, Foc4, Foc5, Foc6, Foc8, Foc10, Foc11, Foc12, Foc 13 and Foc 14 are highly pathogenic nature and other isolates were strongly pathogenic. The cultural variability of these isolates have pale yellowish to dark pinkish (Foc4) in pigmentation with aerial compact mycelial growth within $7-9$ DAI. The morphological characterization all the isolates produced micro, macro-conidia and chlamydospores within $20 \mathrm{DAI}$ and the size of the spores varied from (micro conidia) $5.6 \times 2.5 \mu \mathrm{m}$ (Foc2) to 12.7 $\mathrm{x} 3.1 \mu \mathrm{m}$ (Foc14) and the isolate (Foc4) maximum size of macro conidia in $29.1 \times 4.9 \mu \mathrm{m}$ and mycelial dry weight of $700 \mathrm{mg}$ at $100 \mathrm{ml}$.

\section{Introduction}

Chickpea (Cicer arietnum L.) is a most important grain legume crop of Cicer genus and cultivated throughout the world for its easy available form of edible proteins and vitamins. In India it is cultivated at cool winter (Rabi) season in semi arid tropics by irrigated or rain-fed conditions (Nene et al., 1984). India is largest producer of chickpea in world sharing 65.25 per cent in area and 65.49 per cent in production and is grown on 10.23 million ha area with production 9.88 million tonnes and productivity $967 \mathrm{~kg} / \mathrm{ha}$ (Thaware et al., 2017). Despite the production was reduced due to several biotic and abiotic factors. Chickpea is noticed to be more than 52 pathogens at cropping season (Harware and Nene, 1980; Nene et al., 1984). Among these pathogens $F$. oxysporum f. sp. ciceris 
causing a potential yield loss for both in seed yield and seed weight by wilt about 10 to 15 per cent (Navas-Cortes et al., 2000b; Khilare et al., 2009).

Fusarium oxysporum f. sp. ciceris is ubiquitous soil borne pathogen and providing severe economic losses about $10-40 \%$ in worldwide (Kaiser et al., 1994). Fusarium genus was highly variable nature in survive, growth and infection in all seasons with crop or without also. Because absence of susceptible host it can survive in soil due to their production of resting spores like, micro, macro conidia and chlamydospores for distribution with diverse niches (Leslie and Summerell, 2006; Nelson et al., 1983). Hence traditionally following methods viz., crop rotation, using resistant cultivars, chemical managements is presence with some limitation factors like, location specific pathogen races and wide geographical distribution (Singh et al., 2006). So, the cultural and morphological variability is primary diagnosis for typical identification of different isolates of $F$. oxysporum f. sp. ciceris and classically determining by their virulence ability.

In the present study was carried out for disease prevalence and extensively discriminating the different isolates of $F$. oxysporum f. sp. ciceris by cultural, morphological characterization and virulence ability through grouping it.

\section{Materials and Methods}

\section{Survey and occurrence}

An extensive survey was conducted in major chickpea growing areas of Tamil Nadu during Rabi, 2015. In each districts locations were selected randomly and a total of fifteen locations were selected from four districts for assessment of wilt incidence by $F$. oxysporum f. sp. ciceris. The name of the villages surveyed along with districts given in Table 1. The wilt incidence was calculated by using the following formula and impact of disease incidence grouped into classes like, $(0 \%$ - Nil, $0.1-1.0 \%$ - low, $1.1-20.0 \%$ - moderately high, 20.1-50.0\% - high and $>50.0 \%$ - very high) (Traperos-Casas, 1983).

Per cent disease Incidence $=$

$\frac{\text { Number } \quad \text { of infected plants }}{\text { Total number of plants }} \times 100$

\section{Fungal isolates}

A large systematic collection of fungal isolates were used in this study, consisting of 15 isolates from infected plant samples. The infected root bits were surface sterilized with $1 \%$ sodium hypochlorite for 30 seconds, and subsequently three washings were given with sterile distilled water. Then, they were placed in sterilized Petri dishes containing potato dextrose agar (PDA) medium (Potato $200 \mathrm{~g}$, dextrose $20 \mathrm{~g}$, agar $15 \mathrm{~g}$ and water 1L) and incubated at the laboratory conditions at $25 \pm$ $2^{\circ} \mathrm{C}$ for seven days. Pure culture of the pathogen was obtained by single hyphal tip method (Rangaswami, 2005).

\section{Pathogenic characterization}

Pathogenic ability was assessed by using local cultivar of JAKI-9218 by the method derived by Harware and Nene (1980). The mixtures of sand and chickpea meal $(90 \mathrm{~g}$ sand $+10 \mathrm{~g}$ chickpea meal) in $250 \mathrm{ml}$ conical flasks were inoculated with 5 plugs ( $8 \mathrm{~mm}$ in diameter) from different isolates of $F$. oxysporum $\mathrm{f}$. sp. ciceris well grown on PDA medium containing Petri plates at $23+1^{\circ} \mathrm{C}$ for ten days. Fifteen days after incubation the multiplied fungal mass was well mixed with $2 \mathrm{~kg}$ of autoclaved soil in $15 \mathrm{~cm}$ diameter plastic pots. Five seeds were sown in each pot and kept under $25 \pm 1^{\circ} \mathrm{C}$ with relative humidity of $30-50 \%$. In each isolate three 
replications were maintained and the control was maintained by uninoculated healthy. These were kept in glasshouse conditions for 40 days till flowering. Plants were observed for symptom development and the pathogenic variability given in to five groups viz., $(0 \%$ wilting - Non pathogenic; 1-20\% wilting weakly pathogenic; $21-50 \%$ wiltingmoderately pathogenic; $51-70 \%$ wiltingstrongly pathogenic and $>70 \%$ wilting- highly pathogenic) was described by Rakhonde et al., (2015).

\section{Cultural characterization}

Fifteen isolates of Fusarium oxysporum f. sp. ciceris obtained aseptically and invidually inoculated in PDA contained Petri dishes and incubated at $28 \pm 2^{\circ} \mathrm{C}$ for a week. Observations of cultural characteristics viz., Colony morphology, colony diameter, growth rate, growth habitat, pigmentation and sporulating potential were recorded at two weeks after incubation respectively. The grouping was done in the basis of mycelial growth for Slow (10 mm/day), medium (10-12 $\mathrm{mm} /$ day) and fast (>12 mm /day) denoted by Dubey et al., (2010).

\section{Morphological characterization}

The morphological characteristics of conidial size (Micro and macro conidia) in respect of each test isolate were studied. The size of conidia was measured using ocular micrometer (calibrated using stage micrometer) under the compound microscope (Labomed Vision 2000) at 400X magnification.

\section{Results and Discussion}

Understanding the basic information of pathogen's viz., pathogenic diversity, cultural and morphological variability was most playing a important role in the development of durable resistance. $F$. oxysporum f. sp. ciceris is a highly variable nature on other soil borne pathogens. Because of their survival nature and virulence is known to be a vital role in disease incidence and resistance occurrence in chickpea plants.

\section{Survey and occurrence}

Survey was conducted to assess the incidence of wilt disease in major chickpea growing districts of Tamil Nadu viz., Coimbatore, Dindigul, Dharmapuri and Tiruppur during Rabi, 2015 (Table 1; Plate 1). The result revealed that the maximum disease incidence of 57.33 per cent was recorded at Gomangalampudur in Tiruppur district of cultivar JAKI-9218 and a minimum of 34.00 per cent incidence was recorded at Idigarai in Coimbatore district in $\mathrm{CO} 4$ cultivar (Table 1, Figure 1). These results revealed that Kumar et al., (2012) he reported that the chickpea was attained highly incidence of $F$. oxysporum f. sp. ciceris at 38.79 to 59.23 in Ranchi of Jharkhand state. In 25 to 48 per cent of local cultivars of chickpea in field conditions were influenced by wilt at $9.7 \%$ to $13.8 \%$ in major central and southern parts of India (Ghosh et al., 2013).

\section{Collection of fungal isolates}

An extensive survey was conducted during Rabi, 2015 in major chickpea growing areas of Tamil Nadu. The prevalent incidence of wilt was recorded and the fifteen Fusarium oxysporum f. sp. ciceris isolates were isolated and confirmed through their production of spores and phenotypic appearance of cultural growth.

\section{Pathogenic characterization}

The pathogenic variability test indicates that all the fifteen isolates of Fusarium oxysporum 
f. sp. ciceris, proved to be pathogenic to local cultivar JAKI-9218. The fifteen isolates were grouped under five forms like, (Non pathogenic, weakly pathogenic and moderately pathogenic have no represent isolates). Among the all isolates, there are six isolates viz., Foc1, Foc2, Foc3, Foc7, Foc9 and Foc15 were strongly pathogenic nature and causing wilt incidence ranged from 53.3 $\%$ to $66.7 \%$. Another nine isolates viz., Foc4, Foc5, Foc6, Foc8, Foc10, Foc11, Foc12, Foc13 and Foc14 were recorded incidence at $73.3 \%$ to $93.3 \%$ under highly pathogenic respectively (Table 2 and 5). These results revealed that Sharma et al., (2009) reported forty eight $F$. oxysporum f. sp. ciceris isolates from India, among the forty one isolates have been identified as highly pathogenic and remaining seven isolates were non-pathogenic respectively. The existence of pathogenic variability in $F$. oxysporum f. sp. ciceris isolates was also reported by Gupta et al., (1986), Paul et al., (2001) and Mandhare et al., (2011) from isolated from different regions of India.

Figure 1. Survey for the incidence of wilt in major chickpea growing areas of Tamil Nadu

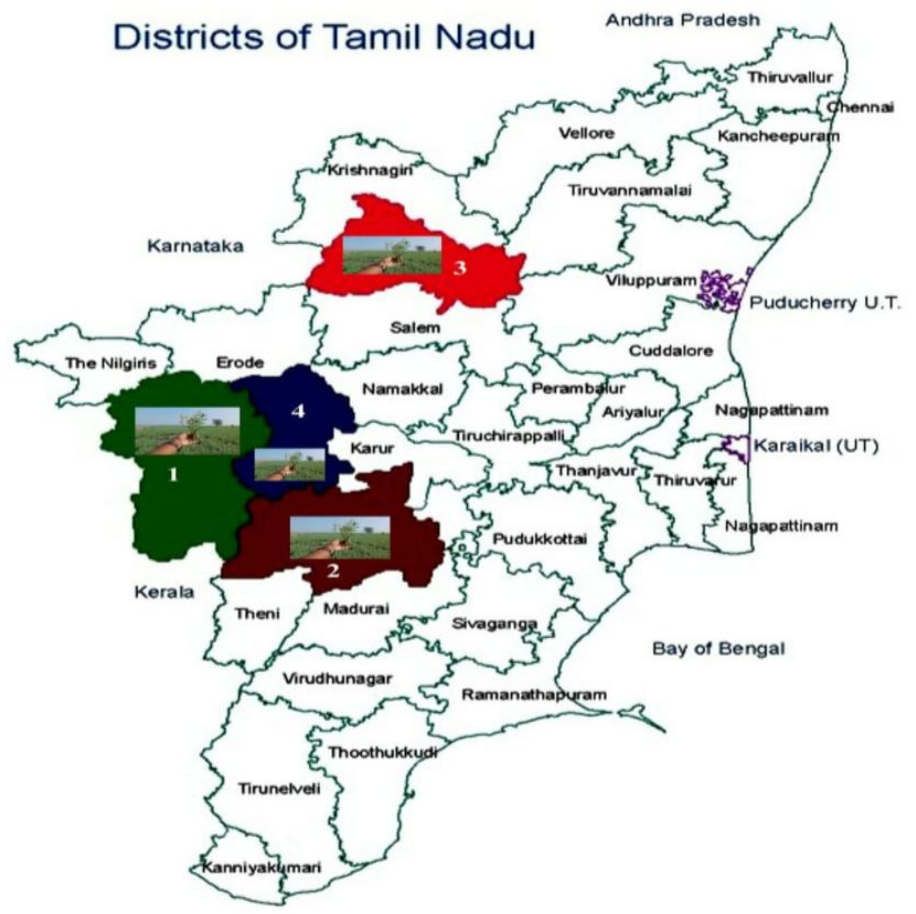

Survey conducted districts
1. Coimbatore
2. Dindigul
3. Dharmapuri
4. Tiruppur 
Figure 2. Different isolates of Fusarium oxysporum f. sp. ciceris from major chickpea growing areas of Tamil Nadu

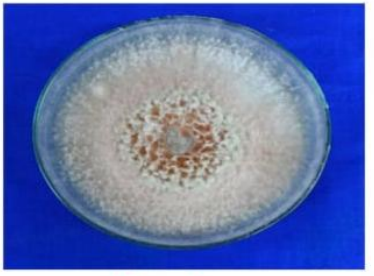

Foc 1

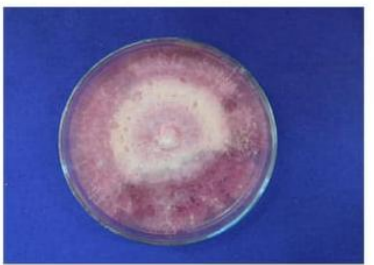

Foc 4

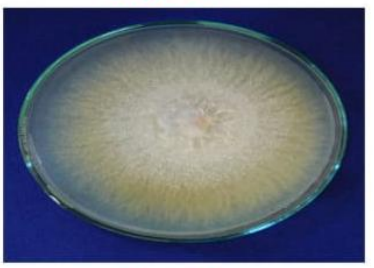

Foc 7

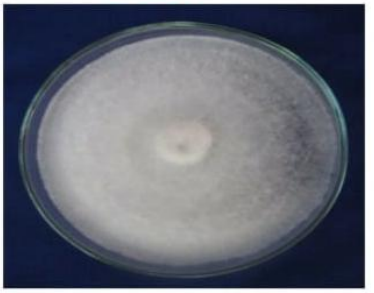

Foc 10

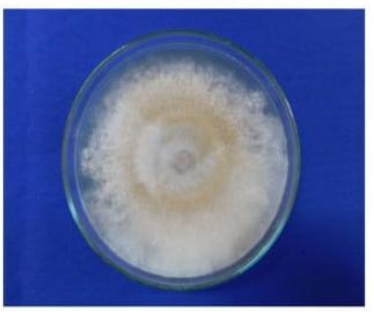

Foc 13

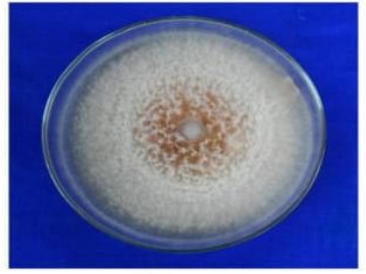

Foc 2

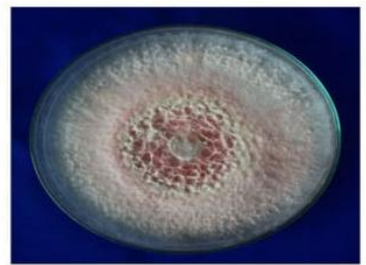

Foc 5

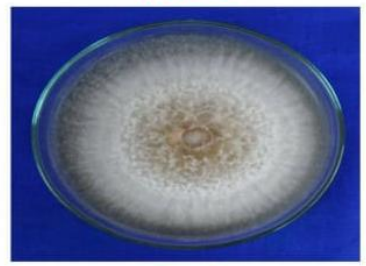

Foc 8

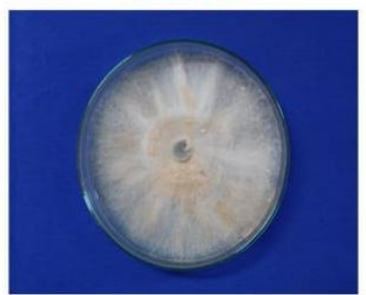

Foc 11

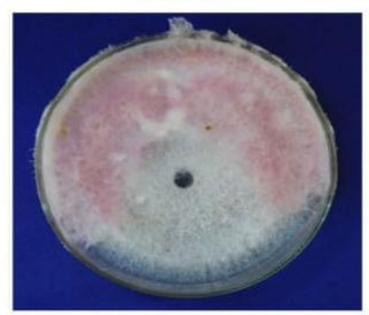

Foc 14

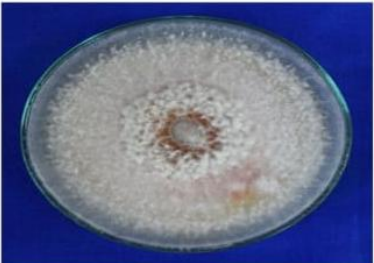

Foc 3

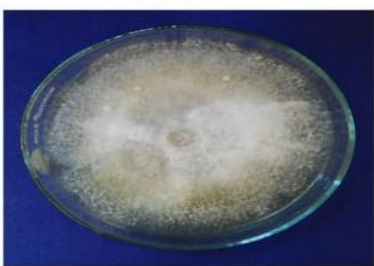

Foc 6

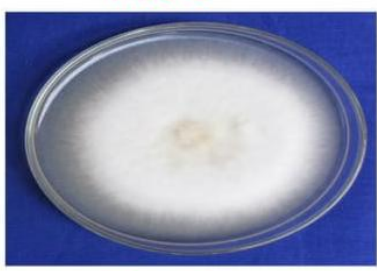

Foc 9

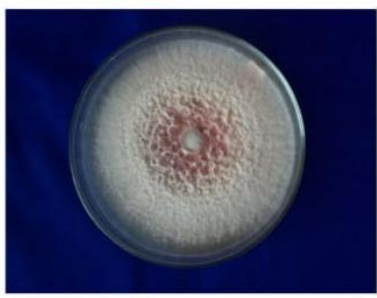

Foc 12

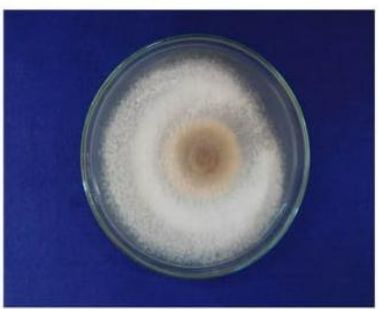

Foc 15

Foc11-Poolankinaru

Foc12- Ragalpavi

Foc13- Ramachandrapuram

Foc14- Thippampatti

Foc15- Valzavadi 
Figure 3. Microscopic view of Fusarium oxysporum f. sp. ciceris isolates

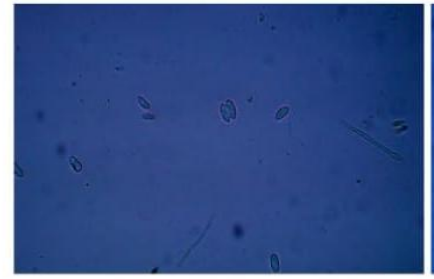

Foc 1

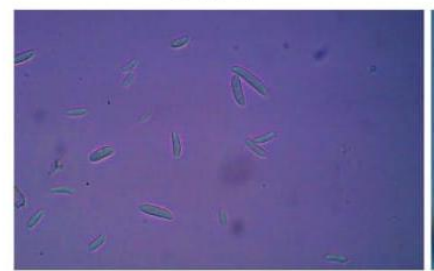

Foc 4

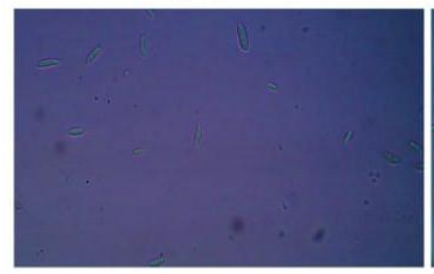

Foc 7

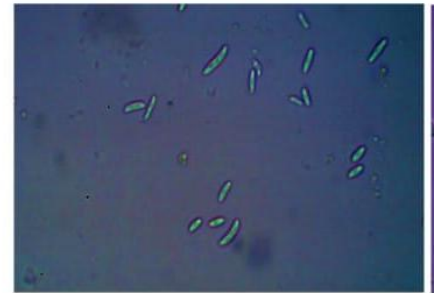

Foc 10

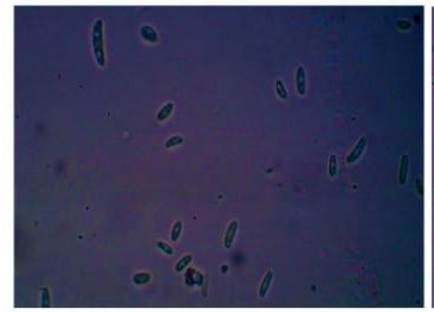

Foc 13

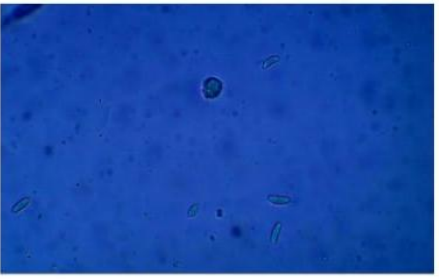

Foc 2

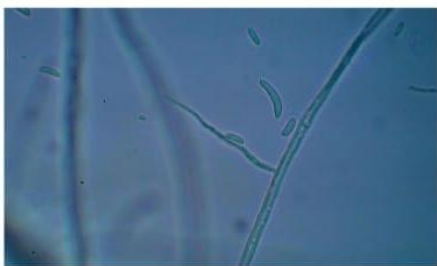

Foc 5

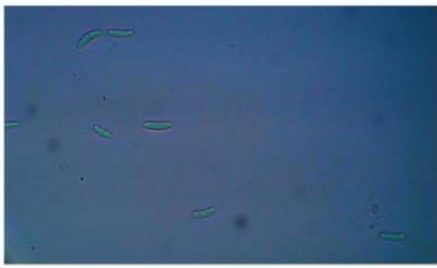

Foc 8

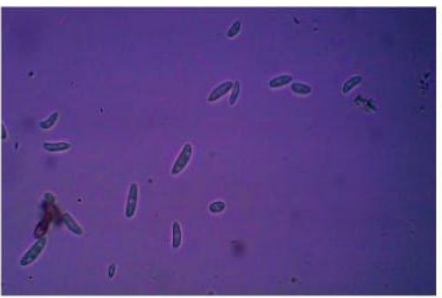

Foc 11

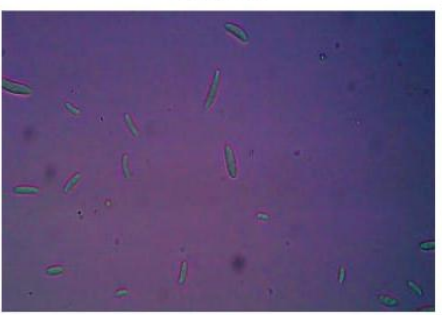

Foc 14

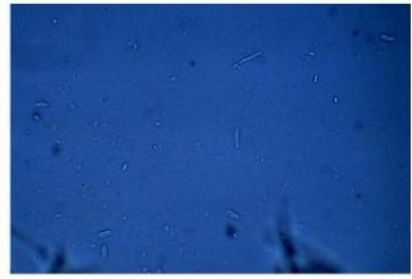

Foc 3

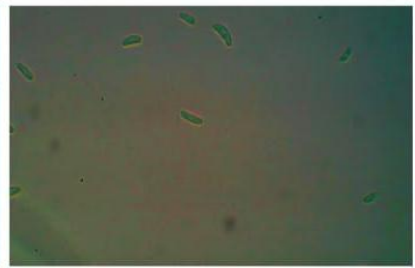

Foc 6

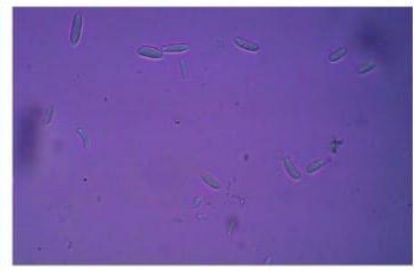

Foc 9

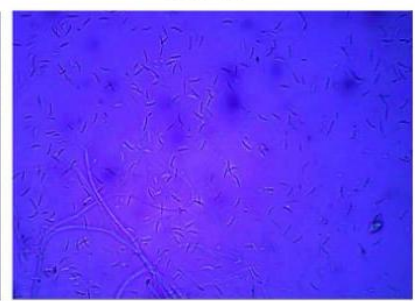

Foc 12

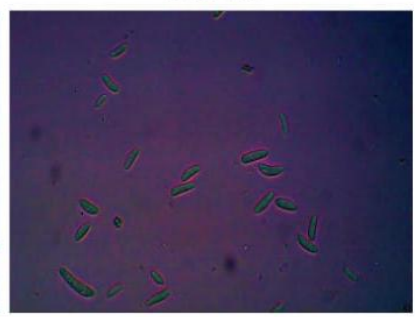

Foc 15 
Table.1 Survey and occurrence of Fusarium wilt disease incidence of major chickpea (C. arietinum L.) growing areas of Tamil Nadu

\begin{tabular}{|c|c|c|c|c|c|c|}
\hline S. No. & Districts & Location & Cultivar & $\begin{array}{c}\text { Isolate } \\
\text { No }\end{array}$ & $\begin{array}{l}\text { Per cent } \\
\text { disease } \\
\text { incidence } \\
\text { (PDI)* }\end{array}$ & Impact \\
\hline \multirow[t]{2}{*}{1} & \multirow[t]{2}{*}{ Coimbatore } & Idigarai & $\mathrm{CO} 4$ & Foc5 & $34.67^{\text {h }}$ & High \\
\hline & & Periyanakanpalayam & $\mathrm{CO} 4$ & Foc10 & $41.00^{\text {def }}$ & High \\
\hline 2 & Dharmapuri & Athakampapu & JAKI -9218 & Foc3 & $35.33^{\text {h }}$ & High \\
\hline 3 & Dindigul & Konnur & JAKI-9218 & Foc6 & $39.67^{\text {defg }}$ & High \\
\hline \multirow{11}{*}{4} & \multirow{11}{*}{ Tiruppur } & Adivalli & JAKI-9218 & Foc1 & $37.00^{\text {gh }}$ & High \\
\hline & & Anthiyur & JAKI-9218 & Foc2 & $38.33^{\text {fg }}$ & High \\
\hline & & Gomangalampudur & JAKI-9218 & Foc4 & $57.33^{\mathrm{a}}$ & $\begin{array}{l}\text { Very } \\
\text { high }\end{array}$ \\
\hline & & Modakkupatti & JAKI-9218 & Foc7 & $42.33^{\text {cd }}$ & High \\
\hline & & Mukkonam & JAKI-9218 & Foc8 & $44.33^{b c}$ & High \\
\hline & & Pannaikinaru & JAKI-9218 & Foc9 & $39.33^{\text {efg }}$ & High \\
\hline & & Ragalpavi & JAKI-9218 & Foc12 & $37.33^{\text {gh }}$ & High \\
\hline & & Ramachandrapuram & JAKI-9218 & Foc13 & $41.33^{\text {de }}$ & High \\
\hline & & Poolankinaru & JAKI-9218 & Foc11 & $40.67^{\text {def }}$ & High \\
\hline & & Thippampatti & JAKI-9218 & Foc14 & $46.33^{b}$ & High \\
\hline & & Valzavadi & JAKI-9218 & Foc15 & $42.33^{\text {cd }}$ & High \\
\hline
\end{tabular}

Means followed by a common letter are not significantly different at the $5 \%$ level by the DMRT. 
Table.2 Pathogenic variability for different isolates of Fusarium oxysporum f. sp. ciceris against with local cultivar JAKI-9218 under in vitro conditions

\begin{tabular}{|c|c|c|c|c|c|}
\hline S. No & Locations & $\begin{array}{c}\text { Isolate } \\
\text { Name }\end{array}$ & $\begin{array}{c}\text { Germination } \\
\mathbf{( \% )}\end{array}$ & $\begin{array}{c}\mathbf{( \% )} \\
\text { Wilting }\end{array}$ & $\begin{array}{c}\text { Type of } \\
\text { pathogenic }\end{array}$ \\
\hline $\mathbf{1}$ & Adivalli & Foc1 & 80.0 & 53.3 & SP \\
\hline $\mathbf{2}$ & Anthiyur & Foc2 & 86.7 & 53.3 & SP \\
\hline $\mathbf{3}$ & Athakkampapu & Foc3 & 86.7 & 60.0 & SP \\
\hline $\mathbf{4}$ & Gomangalampudur & Foc4 & 100.0 & 93.3 & HP \\
\hline $\mathbf{5}$ & Idigarai & Foc5 & 86.7 & 73.3 & HP \\
\hline $\mathbf{6}$ & Konnur & Foc6 & 93.3 & 80.0 & HP \\
\hline $\mathbf{7}$ & Modakkupatti & Foc7 & 93.3 & 60.0 & SP \\
\hline $\mathbf{8}$ & Mukkonam & Foc8 & 100.0 & 73.3 & HP \\
\hline $\mathbf{9}$ & Pannaikinaru & Foc9 & 80.0 & 66.7 & SP \\
\hline $\mathbf{1 0}$ & Periyanakanpalayam & Foc10 & 93.3 & 73.3 & HP \\
\hline $\mathbf{1 1}$ & Poolankinaru & Foc11 & 100.0 & 73.3 & HP \\
\hline $\mathbf{1 2}$ & Ragalpavi & Foc12 & 100.0 & 80.0 & HP \\
\hline $\mathbf{1 3}$ & Ramachandrapuram & Foc13 & 100.0 & 80.0 & HP \\
\hline $\mathbf{1 4}$ & Thippampatti & Foc14 & 93.3 & 86.7 & HP \\
\hline $\mathbf{1 5}$ & Valzavadi & Foc15 & 93.3 & 66.7 & SP \\
\hline $\mathbf{1 6}$ & Control & Uninoculated & 100.0 & 0.00 & NIL \\
\hline
\end{tabular}


Table.3 Cultural characteristics for different isolates of $F$. oxysporum f. sp. ciceris

\begin{tabular}{|c|c|c|c|c|c|c|c|c|}
\hline S. No & Isolate No & $\begin{array}{c}\text { Colony } \\
\text { morphology }\end{array}$ & $\begin{array}{c}\text { Mean } \\
\text { mycelial } \\
\text { growth }(\mathbf{m m}) / \\
\text { 7DAI }\end{array}$ & $\begin{array}{c}\text { Growth rate } \\
(\mathrm{mm})\end{array}$ & $\begin{array}{l}\text { Growth } \\
\text { habitat }\end{array}$ & $\begin{array}{l}\text { No. of. days } \\
\text { taken to } \\
\text { cover the } \\
\text { plate }\end{array}$ & Pigmentation & Sporulation \\
\hline 1. & Foc 1 & $\begin{array}{c}\text { Circular } \\
\text { compact aerial } \\
\text { mycelia }\end{array}$ & $\begin{array}{c}80.00 \\
(63.43)\end{array}$ & $11.42^{\text {de }}$ & Medium & 8 & Pale pinkish & $\begin{array}{l}\text { 1-2 celled } \\
\text { sparsely } \\
\text { dispersed } \\
\text { microconidia }\end{array}$ \\
\hline 2. & Foc 2 & $\begin{array}{c}\text { Smooth circular } \\
\text { compact aerial } \\
\text { mycelia }\end{array}$ & $\begin{array}{c}77.00 \\
(61.34)\end{array}$ & $11.00^{f}$ & Medium & 9 & $\begin{array}{l}\text { Pale pinkish } \\
\text { with white }\end{array}$ & $\begin{array}{c}\text { Sparsely } \\
\text { dispersed } \\
\text { microconidia } \\
\text { with minimum } \\
\text { curvature }\end{array}$ \\
\hline 3. & Foc 3 & $\begin{array}{c}\text { Circular } \\
\text { profuse } \\
\text { compact aerial } \\
\text { mycelia }\end{array}$ & $\begin{array}{c}74.00 \\
(59.34)\end{array}$ & $10.57^{\mathrm{g}}$ & Medium & 9 & $\begin{array}{l}\text { Pale pinkish } \\
\text { with white }\end{array}$ & $\begin{array}{c}\text { Sparsely } \\
\text { dispersed } \\
\text { microconidia }\end{array}$ \\
\hline 4. & Foc 4 & $\begin{array}{c}\text { Circular } \\
\text { compact } \\
\text { 5.aerial mycelia }\end{array}$ & $\begin{array}{c}90.00 \\
(71.56)\end{array}$ & $12.85^{\mathrm{a}}$ & Fast & 7 & Deep pinkish & $\begin{array}{c}\text { Abundantly } \\
\text { dispersed micro } \\
\text { and 3-5 septate } \\
\text { macroconidia }\end{array}$ \\
\hline 5. & Foc 5 & $\begin{array}{c}\text { Circular smooth } \\
\text { compact aerial } \\
\text { mycelia }\end{array}$ & $\begin{array}{c}77.00 \\
(61.34)\end{array}$ & $11.00^{\mathrm{f}}$ & Medium & 9 & Pale pinkish & $\begin{array}{c}\text { Sparsely } \\
\text { dispersed } \\
\text { microconidia } \\
\text { and } \\
\text { macroconidia }\end{array}$ \\
\hline 6. & Foc 6 & $\begin{array}{c}\text { Circular } \\
\text { sparsely dense } \\
\text { aerial mycelia }\end{array}$ & $\begin{array}{c}78.67 \\
(62.48)\end{array}$ & $11.23^{\mathrm{e}}$ & Medium & 9 & Pale yellowish & $\begin{array}{l}\text { 1-2 celled } \\
\text { sparsely } \\
\text { dispersed } \\
\text { microconidia }\end{array}$ \\
\hline 7. & Foc 7 & $\begin{array}{l}\text { Circular } \\
\text { sparsely } \\
\text { flattened } \\
\text { mycelia }\end{array}$ & $\begin{array}{c}80.67 \\
(63.91)\end{array}$ & $11.52^{d}$ & Medium & 8 & $\begin{array}{l}\text { Yellowish with } \\
\text { centre white }\end{array}$ & $\begin{array}{l}\text { 1-2 celled } \\
\text { Abundantly } \\
\text { dispersed } \\
\text { microconidia }\end{array}$ \\
\hline 8. & Foc 8 & $\begin{array}{c}\text { Circular smooth } \\
\text { compact aerial }\end{array}$ & $\begin{array}{c}83.00 \\
(65.65)\end{array}$ & $11.85^{\mathrm{c}}$ & Medium & 8 & Pale white & $\begin{array}{c}\text { Sparsely } \\
\text { dispersed 1-2 } \\
\text { celled }\end{array}$ \\
\hline
\end{tabular}


Int.J.Curr.Microbiol.App.Sci (2018) 7(2): 1301-1313

\begin{tabular}{|c|c|c|c|c|c|c|c|c|}
\hline & & mycelia & & & & & & microconidia \\
\hline 9. & Foc 9 & $\begin{array}{c}\text { Circular smooth } \\
\text { dense mycelia }\end{array}$ & $\begin{array}{c}75.33 \\
(60.22)\end{array}$ & $10.76^{\mathrm{g}}$ & Medium & 9 & Milky white & $\begin{array}{l}\text { Abundantly } \\
\text { dispersed } \\
\text { microconidia }\end{array}$ \\
\hline 10. & Foc 10 & $\begin{array}{c}\text { Circular smooth } \\
\text { compact } \\
\text { mycelia }\end{array}$ & $\begin{array}{c}69.67 \\
(56.58)\end{array}$ & $9.95^{\mathrm{hi}}$ & Slow & 10 & Milky white & $\begin{array}{l}\text { Abundantly } \\
\text { dispersed } \\
\text { microconidia } \\
\text { and 3-5 celled } \\
\text { macroconidia }\end{array}$ \\
\hline 11. & Foc 11 & $\begin{array}{c}\text { Circular } \\
\text { sparsely dense } \\
\text { flattened } \\
\text { mycelia }\end{array}$ & $\begin{array}{c}70.33 \\
(57.62)\end{array}$ & $10.04^{h}$ & Medium & 10 & Pale yellowish & $\begin{array}{c}\text { Abundantly } \\
\text { dispersed 1-2 } \\
\text { celled } \\
\text { microconidia } \\
\text { with minimum } \\
\text { curvature }\end{array}$ \\
\hline 12. & Foc 12 & $\begin{array}{c}\text { Smooth circular } \\
\text { compact } \\
\text { mycelia }\end{array}$ & $\begin{array}{c}71.33 \\
(57.62)\end{array}$ & $10.19^{h}$ & Medium & 10 & Pale pinkish & $\begin{array}{c}\text { Abundantly } \\
\text { dispersed 3-5 } \\
\text { celled } \\
\text { macroconidia }\end{array}$ \\
\hline 13. & Foc 13 & $\begin{array}{c}\text { Circular smooth } \\
\text { sparsely dense } \\
\text { mycelia }\end{array}$ & $\begin{array}{c}70.33 \\
(56.99)\end{array}$ & $10.04^{h}$ & Medium & 10 & Pale yellowish & $\begin{array}{c}\text { Abundantly } \\
\text { dispersed 1-2 } \\
\text { celled } \\
\text { microconidia }\end{array}$ \\
\hline 14. & Foc 14 & $\begin{array}{c}\text { Circular } \\
\text { compact aerial } \\
\text { mycelia }\end{array}$ & $\begin{array}{c}86.00 \\
(68.05)\end{array}$ & $12.28^{\mathbf{b}}$ & Fast & 8 & Deep pinkish & $\begin{array}{l}\text { Abundantly } \\
\text { dispersed } 3 \text { - } \\
\text { celled } \\
\text { macroconidia }\end{array}$ \\
\hline 15. & Foc 15 & $\begin{array}{c}\text { Circular smooth } \\
\text { compact aerial } \\
\text { mycelia }\end{array}$ & $\begin{array}{c}68.33 \\
(55.62)\end{array}$ & $9.76^{\mathrm{i}}$ & Slow & 10 & $\begin{array}{l}\text { Milky white } \\
\text { with pale } \\
\text { yellowish }\end{array}$ & $\begin{array}{l}\text { Abundantly } \\
\text { dispersed } \\
\text { microconidia }\end{array}$ \\
\hline
\end{tabular}


Table.4 Conidial characteristics for different isolates of $F$. oxysporum $\mathrm{f}$. sp. ciceris

\begin{tabular}{|c|c|c|c|c|c|c|c|}
\hline \multirow[t]{2}{*}{ S. No. } & \multirow{2}{*}{$\begin{array}{c}\text { Isolate } \\
\text { No }\end{array}$} & \multicolumn{2}{|c|}{ Microconidia } & \multicolumn{2}{|c|}{ Macroconia } & \multirow{2}{*}{$\begin{array}{c}\text { No of } \\
\text { conidia / } \\
\mu \mathrm{L}\end{array}$} & \multirow{2}{*}{$\begin{array}{c}\text { Mycelial dry } \\
\text { weight }(\mathrm{mg}) / \\
100 \mathrm{ml}^{*}\end{array}$} \\
\hline & & $\begin{array}{c}\text { Length } \\
(\mu \mathrm{m})\end{array}$ & $\begin{array}{l}\text { Width } \\
(\mu \mathrm{m})\end{array}$ & $\begin{array}{c}\text { Length } \\
(\mu \mathrm{m})\end{array}$ & $\begin{array}{c}\text { Width } \\
(\mu \mathrm{m})\end{array}$ & & \\
\hline 1. & Foc 1 & 6.5 & 2.6 & 16.9 & 4.1 & 8 & $553^{\mathrm{hi}}$ \\
\hline 2. & Foc 2 & 5.6 & 2.5 & 17.3 & 3.8 & 5 & $550^{\mathrm{fg}}$ \\
\hline 3. & Foc 3 & 6.9 & 2.7 & 18.5 & 4.1 & 5 & $540^{\text {gh }}$ \\
\hline 4. & Foc 4 & 11.7 & 2.9 & 29.1 & 4.9 & 18 & $700^{b}$ \\
\hline 5. & Foc 5 & 10.5 & 2.7 & 20.2 & 3.8 & 7 & $653^{d}$ \\
\hline 6. & Foc 6 & 11.3 & 2.8 & 21.2 & 4.4 & 9 & $663^{d}$ \\
\hline 7. & Foc 7 & 8.1 & 2.6 & 20.5 & 4.2 & 15 & $535^{\mathrm{hi}}$ \\
\hline 8. & Foc 8 & 8.8 & 2.8 & 22.9 & 4.1 & 7 & $678^{c}$ \\
\hline 9. & Foc 9 & 10.9 & 2.6 & 24.9 & 4.3 & 15 & $528^{\mathrm{ij}}$ \\
\hline 10. & Foc 10 & 7.8 & 2.6 & 28.4 & 4.4 & 17 & $522^{\mathbf{j}}$ \\
\hline 11. & Foc 11 & 8.1 & 2.7 & 20.6 & 3.8 & 18 & $597^{e}$ \\
\hline 12. & Foc 12 & 10.9 & 3.0 & 25.6 & 4.5 & 70 & $551^{f}$ \\
\hline 13. & Foc 13 & 10.1 & 2.6 & 22.0 & 4.4 & 18 & $496^{k}$ \\
\hline 14. & Foc 14 & 12.7 & 3.1 & 23.6 & 4.4 & 18 & $723^{a}$ \\
\hline 15. & Foc 15 & 11.0 & 2.7 & 26.3 & 4.1 & 22 & $492^{k}$ \\
\hline
\end{tabular}

* Mean of three replications

Means followed by a common letter are not significantly different at the $5 \%$ level by DMRT.

Table.5 Grouping of F. oxysporum f. sp. ciceris isolates on the basis of pathogenic nature and growth habitat

\begin{tabular}{|c|c|c|c|c|}
\hline \multirow[t]{2}{*}{ S. No } & \multicolumn{2}{|c|}{ Pathogenic Nature } & \multicolumn{2}{|c|}{ Growth Habitat } \\
\hline & Group ((\% wilting) & Name of the isolates & $\begin{array}{c}\text { Group (Colony } \\
\text { diameter mm/day) }\end{array}$ & $\begin{array}{c}\text { Name of the } \\
\text { isolates }\end{array}$ \\
\hline 1. & Non-pathogenic $(0 \%)$ & Nil & Slow (10mm /day) & $\begin{array}{l}\text { Foc10 and } \\
\text { Foc15 }\end{array}$ \\
\hline 2. & $\begin{array}{c}\text { Weakly pathogenic (1- } \\
20 \%)\end{array}$ & Nil & $\begin{array}{l}\text { Medium (>10- } \\
12 \mathrm{~mm} / \text { day) }\end{array}$ & $\begin{array}{l}\text { Foc1, Fo } 2 \\
\text { Foc } 3 \text {, Foc5, } \\
\text { Foc6, Foc7, } \\
\text { Foc8, Foc9, } \\
\text { Foc11, Foc12 } \\
\text { and Foc13 }\end{array}$ \\
\hline 3. & $\begin{array}{l}\text { Moderately pathogenic } \\
\qquad(21-50 \%)\end{array}$ & Nil & Fast (>12mm / day) & $\begin{array}{l}\text { Foc4 and } \\
\text { Foc14 }\end{array}$ \\
\hline 4. & $\begin{array}{l}\text { Strongly pathogenic } \\
(51-70 \%)\end{array}$ & $\begin{array}{c}\text { Foc1, Foc } 2, \text { Foc3, } \\
\text { Foc7, Foc9 and Foc15 }\end{array}$ & & \\
\hline 5. & $\begin{array}{l}\text { Highly pathogenic } \\
\qquad(>70 \%)\end{array}$ & $\begin{array}{l}\text { Foc4, Foc5, Foc6, } \\
\text { Foc8, Foc10, Foc11, } \\
\text { Foc12, Foc13 and } \\
\text { Foc14 }\end{array}$ & & \\
\hline
\end{tabular}




\section{Cultural characterization}

All the fifteen isolates of Fusarium oxysporum f. sp. ciceris exhibited a high variability in colony morphology, colony diameter, growth rate, growth habitat, pigmentation and sporulating potential respectively. The colony morphology varied from compact dense aerial mycelia to sparsely flattened mycelia of pale yellowish to deep pinkish coloured with slow to fast growth habitat. Among the fifteen isolates (Foc4) recorded a three types of growth like, slow (Foc10 and Foc15), medium (Foc1, Foc2, Foc3, Foc5, Foc6, Foc7, Foc8, Foc9, Foc11, Foc12 and Foc13), fast (Foc4 and Foc14) are recorded and abundantly production of micro and macro conidia in 7 DAI (Day after inoculation) (Table 3 and 5, Figure 2). These results are coincided with earlier workers like, Gupta et al., (1986), Desai et al., (1994). Burgess et al., (1989) reported that the Fusarium oxysporum were extensively variable of cultural and morphological diversity and it's concluded for identification of genus not species.

\section{Morphological characterization}

All the isolates were highly variable in morphological viz., production of micro, macro conidia and chlamydospores. The mean size of micro conidia of the test isolates ranged from $5.6 \times 2.5 \mu \mathrm{m}$ (Foc2) to $12.7 \times 3.1$ $\mu \mathrm{m}$ (Foc14) and the isolate (Foc4) maximum size of macro conidia in $29.1 \times 4.9 \mu \mathrm{m}$ and mycelial dry weight of $700 \mathrm{mg}$ in $100 \mathrm{ml}$ and highly pathogenic also (Table 4 and Figure 3). Above the results were revealed that Dubey et al., (2010) documented one hundred and twelve isolates by twelve categories, among the isolates were produced micro conidia size varied from 5.1-12.8 $\times$ 2.5-5.0 $\mu \mathrm{m}$ and macro conidia $(16.5-37.9 \times 4.0-5.9 \mu \mathrm{m})$ with $1-5$ septations. Kaur et al., (2015) reported twenty four isolates of Fusarium oxysporum f. sp. ciceris produced significant variation to size of micro $(8.9-16.9 \times 3.1-6.3 \mu \mathrm{m})$ and macro $(21.7-64.9 \times 2.7-10.0 \mu \mathrm{m})$ conidia was observed also.

In conclusion, collection of different isolates of $F$. oxysporum f. sp. ciceris was highly variable nature through their presence pathogenic nature and growth habitat and cultural characters also. Because the virulent isolate of $F$. oxysporum f. sp. ciceris (Foc4) growing fast and production of spores abundantly within seven days and their causing severe incidence in local cultivar of JAKI-9218 in Tamil Nadu.

\section{Acknowledgements}

DST- FIST Lab, Department of Plant Pathology, Tamil Nadu Agricultural University, Coimbatore, India.

\section{References}

Burgess, L. W., Nelson, P. E. and Summerell, B. A. 1989. Variability and stability for morphological characters of Fusarium oxysporum isolated from soils in Australia. Mycologia., 81: 818-822.

Desai, S., Nene, Y. L. and Reddy, R. 1994. Races of Fusarium oxysporum causing wilt in chickpea growth variability. Indian J. Mycol Pl Pathol., 24: 120-127.

Díaz, R. M. 1994. Screening of wild Cicer species for resistance to races 0 and 5 of

Dubey, S. C., Singh, S. R. and Singh, B. 2010. Morphological and pathogenic variability of Indian isolates of Fusarium oxysporum f. sp. ciceris causing chickpea wilt. Archives of Phytopathol and Plant Prot., 43: 174-189.

Ghosh, R., Sharma, M., Telangre, R. and Pande, S. 2013. Occurrence and distribution of chickpea diseases in central and southern parts of India. American J. Plant Sci., 4: 940-944.

Gupta, O. M., Khare, M. N. and Kotasthane, S. 
R. 1986. Variability among six isolates of Fusarium oxysporum f. sp. ciceris causing vascular wilt of chickpea. Ind Phytopathol., 39: 279-281.

Kumar, A., Lal, H. C. and Akhtar, J. 2012. Morphological and pathogenic characterization of Fusarium oxysporum f. sp. ciceris causing wilt of chickpea. Ind Phytopath., 65(1): 64-66.

Harware, M. P. and Nene, Y. L. 1980. Influence of wilt at different stages on the yield loss in chickpea. Trop Grain Legume Bull., 19: 38-40.

Kaiser, W. J., Alcala- Jimenez, A. R., HervasVargas, A., Trapero-Casas, J. L. and Jimenez- Diaz, R. M. 1994. Screening of wilt Cicer species for resistance to race 0 and 5 of Fusarium oxysporum f. sp. ciceris. Plant Dis., 78: 962-967.

Kaur, A., Sharma, V. K., Sirari, A., Kaur, J., Singh, G. and Kumar, P. 2015. Variability in Fusarium oxysporum f. sp. ciceris causing wilt in chickpea. African $J$. Microbiol Res., 9 (15): 1089-1097.

Khilare, V. C., Ahmed, R., Chavan, S. S. and Kohire, O. D. 2009. Management of Fusarium oxysporum f. sp. ciceris by different fungicides. Bioinfolet., 6: 41-43.

Leslie, J. F. and Summerell, B. A. 2006. The Fusarium, Laboratory Manual, Blackwell Publishing. pp. 1-388.

Mandhare, V. K., Deshmukh, G. P., Patil, J. V., Kale, A. A. and Chavan, U. D. 2011. Morphological, pathogenic and molecular characterization of Fusarium oxysporum f. sp. ciceris isolates from Maharashtra, India. Indonesean J Agrl Sci., 12(2): 4756.

Navas-Cortes, J. A., Hau, B. and Jimenez-Díaz, R. M. 2000b. Yield loss in chickpeas in relation to development of Fusarium wilt epidemics. Phytopathol., 90:1269-1278.
Nene, Y. L., Sheila, V. K. and Sharma, S. B. 1984. A world list of chickpea (Cicer arietinum L.) and pigeonpea (Cajanus cajan L.) pathogens. Pulse progess report. ICRISAT, Patencheru. India. 32:19.

Paul, J., Gill, T. S. and Singh, R. S. 2001. Variability among isolates of Fusarium oxysporum f. sp. ciceris from chickpea roots and rhizosphere. Plant Disease Res., 16: 116-118.

Rakhonde, P. N., Mane, S. S., Gawande, A. D., Bangar, S. S. and Moharil, M. P. 2015. Molecular and pathogenic variability among Indian isolates of Fusarium oxysporum

f. sp. ciceris causing wilt in chickpea. $J$ Envir. Sci., 7: 21-28.

Rangaswami, G. 2005. Diseases of crop plants in India. Prentice Hall of India Pvt. Ltd. New Delhi. pp. 520.

Sharma, M., Varshney, R. K., Narayanan Rao, J., Kannan, S., Hoisington, D. and Pande, S. 2009. Genetic diversity in Indian isolates of Fusarium oxysporum f. sp. ciceris, chickpea wilt pathogen. African J. Biotechnol., 8 (6): 1016-1023.

Singh, B. P., Saikia, R., Yadav, M., Singh, R., Chauhan, V. S. and Arora, D. K. 2006. Molecular characterization of Fusarium oxysporum f. sp. ciceris causing wilt of chickpea. Afr J. Biotechnol., 5:497-502.

Thaware, D. S., Kohire, O. D. and Gholve, V. M. 2017. In vitro efficacy of fungal and bacterial antagonists against Fusarium oxysporum f. sp. ciceris causing chickpea wilt. Int J. Current Microbiol. Appl Sci., 6(1): 905-909.

Trapero-Casas, A. 1983. Wilt and root rot of chickpea in the Guadalquivir valley: importance, distribution, etiology, epidemiology and control. Ph.D., Thesis, University of Cordoba, Spain. p. 295.

\section{How to cite this article:}

Murali Sankar, P., S. Vanitha, A. Kamalakannan, P. Anantha Raju and Jeyakumar, P. 2018. Prevalence of Fusarium oxysporum f. sp. ciceris Causing Wilt in Chickpea and Its Pathogenic, Cultural and Morphological Characterization. Int.J.Curr.Microbiol.App.Sci. 7(02): 1301-1313. doi: https://doi.org/10.20546/ijcmas.2018.702.159 